\title{
High-resolution mapping of R loops
}

Two novel techniques refine genome-wide mapping of $\mathrm{R}$ loops.

The $\mathrm{R}$ loop is a nucleic acid ménage à trois in which RNA inserts between the two DNA strands, hybridizing with one and forcing the other to bulge out. Identifying the genomic location of $\mathrm{R}$ loops is important for understanding their function, but, current mapping methods offer limited resolution.

Now, Samie Jaffrey's group at Cornell University and Xiang-Dong Fu's laboratory at the University of California, San Diego have generated the two most precise R-loopmapping techniques to date.

The Jaffrey group modified the DNARNA immunoprecipitation sequencing (DRIP-seq) technique, based on antibodymediated capture of RNA-DNA hybrids from fragmented genomic DNA, followed by deep sequencing (Dumelie and Jaffrey, 2017). The resolution of DRIP-seq is limited by the size of immunoprecipitated DNA fragments. Thus, the researchers introduced a step preceding DRIP-seq that relies on cytosine-to-uracil conversion in genomic regions with single-stranded DNA. One DNA strand within the $\mathrm{R}$ loop is single stranded; thus, the R loop can be precisely mapped because of the exclusive presence of uracils. The results confirmed previous findings that most $\mathrm{R}$ loops are associated with promoters and suggested that the boundaries of $\mathrm{R}$ loops are defined by transcription start sites (TSSs) at the $5^{\prime}$ end and the first exon-intron junction at the $3^{\prime}$ end. In intronless genes, which are strongly associated with R loops, the TSS marks the $5^{\prime}$-R-loop border, whereas the $3^{\prime}$ boundaries are variable.

The R-ChIP approach developed by the Fu laboratory relies on the expression of a V5-tagged catalytically dead RNase H, which binds to but does not degrade the RNA in RNA-DNA hybrids (Chen et al.,
2017). The RNA-DNA hybrids are captured by the anti-V5 antibody, followed by chromatin immunoprecipitation. In agreement with previous findings, the R-ChIP experiments showed that most $\mathrm{R}$ loops map to active gene promoters and detected a greater association with open chromatin. The analyses also suggested that R-loop formation requires a nearby free RNA end and correlates with RNA polymerase II pausing at TSSs.

These new tools for R-loop mapping may provide exciting new insights into the (patho)physiological role of $\mathrm{R}$ loops.

\section{Vesna Todorovic}

\section{RESEARCH PAPERS}

Chen, $\mathrm{L}$. et al. R-ChIP using inactive RNase $\mathrm{H}$ reveals dynamic coupling of R-loops with transcriptional pausing at gene promoters. Mol. Cell 68, 745-747.e5 (2017).

Dumelie, J. G. \& Jaffrey, S. R. Defining the location of promoter-associated R-loops at near-nucleotide resolution using bisDRIP-seq. elife 6, e28306 (2017). 